Supporting Information

\title{
Thermoresponsive Chitosan/DOPA-Based Hydrogel as an Injectable Therapy Approach for Tissue-Adhesion and Hemostasis
}

Yufeng Shou, Jiahui Zhang, Shifeng Yan, Pengfei Xia, Pengliang Xu, Guifei Li, Kunxi Zhang,* and Jingbo Yin*

Department of Polymer Materials, School of Materials Science and Engineering, Shanghai University, Shanghai 200444, China

*Corresponding authors: zhangkunxi@,shu.edu.cn (K.Z.); jbyin@,oa.shu.edu.cn (J.Y.) 


\section{Content}

Figure S1 Schematic diagram of measurement to test the tissue-adhesion ability of HBCS-C hydrogels on the surfaces of two porcine skins ................. S3

Figure S2 Solubility contrast of $5 \mathrm{wt} \% \mathrm{CS}, \mathrm{HBCS}$, and HBCS-C in deionized water .. S3

Figure S3 Micro-morphology of $5 \mathrm{wt} \%$ HBCS and HBCS-C hydrogels ............... S4

Figure S4 Schematic representation of thermoresponsive mechanism of HBCS-C

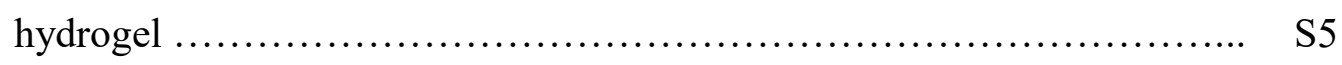

Figure S5 Tissue adhesive behavior of HBCS-C hydrogels, which showed two chicken bones were jointed by HBCS-C hydrogel (5 wt \%) due to its wellperformed adhesion ................................................ S6

Figure S6 Degradation in vitro of HBCS-C hydrogels in PBS and L-PBS $(1 \mathrm{mg} / \mathrm{mL}$

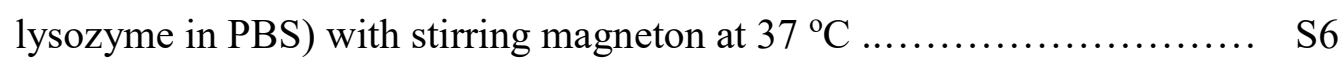

Table S1 Grafting ratio and $\mathrm{M}_{\mathrm{V}}$ of HBCS-C at different molar feed ratios of DOPAC

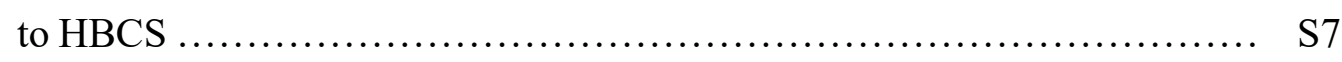



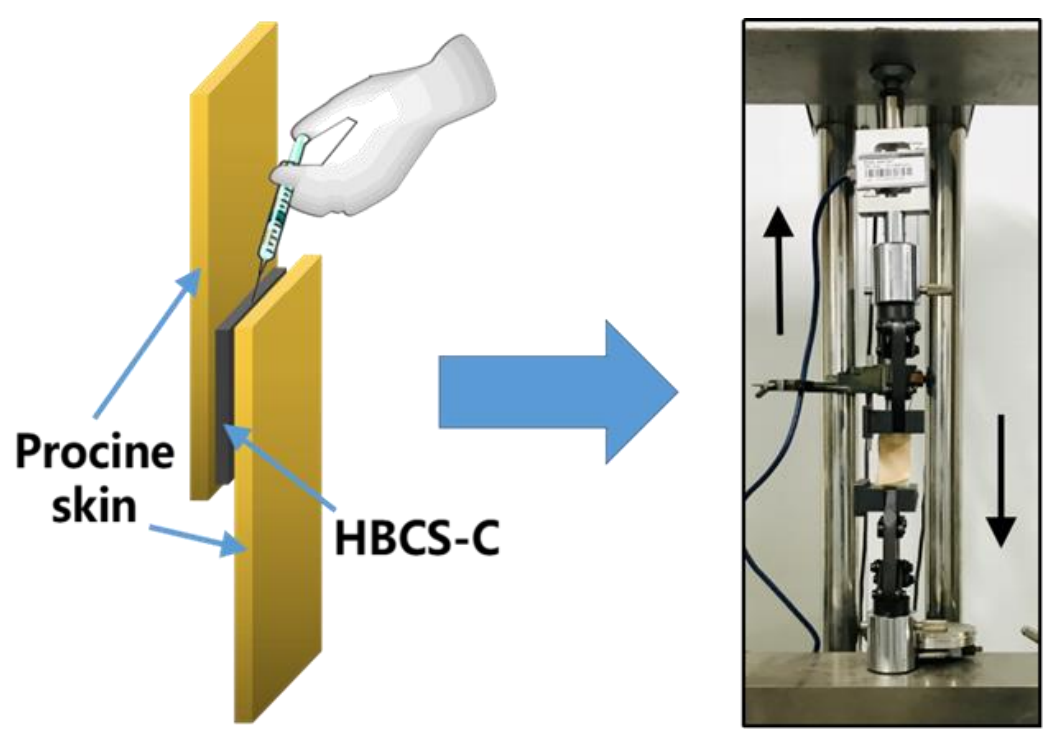

Figure S1. Schematic diagram of measurement to test the tissue-adhesion ability of HBCS-C hydrogels on the surfaces of two porcine skins.

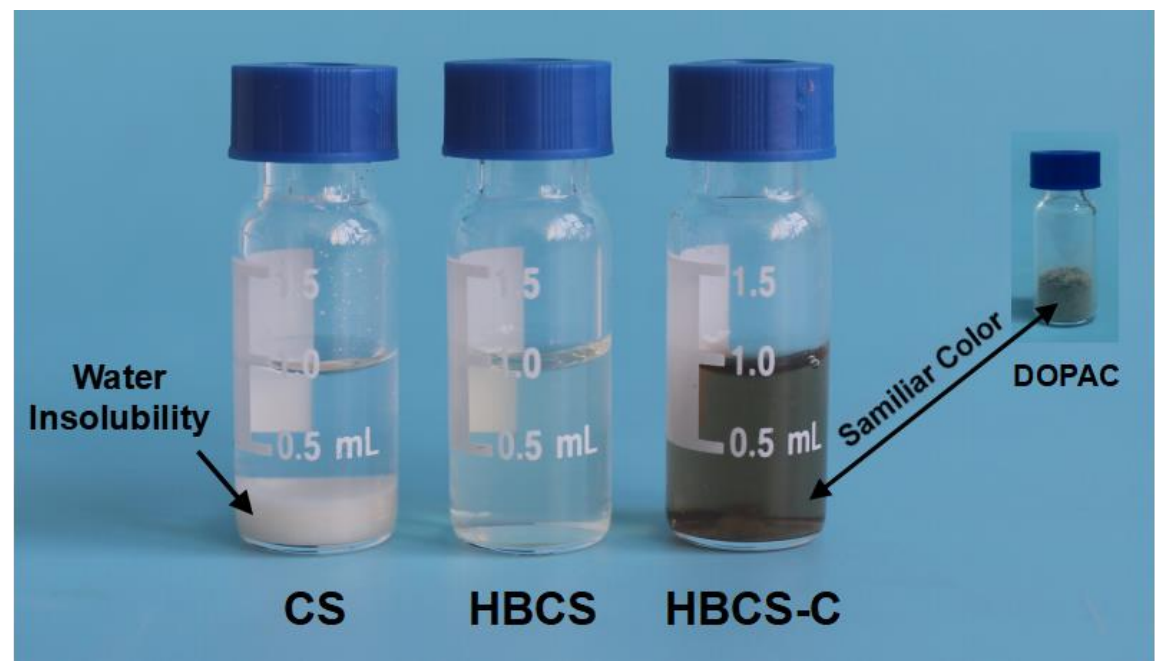

Figure S2. Solubility contrast of $5 \mathrm{wt} \% \mathrm{CS}$, HBCS, and HBCS-C in deionized water. CS powder was insoluble and sank on the bottom of vial, while HBCS and HBCS-C could dissolve in deionized water. The color of HBCS$\mathrm{C}$ solution was similar with the DOPAC (i.e., brown powder). 
(a)

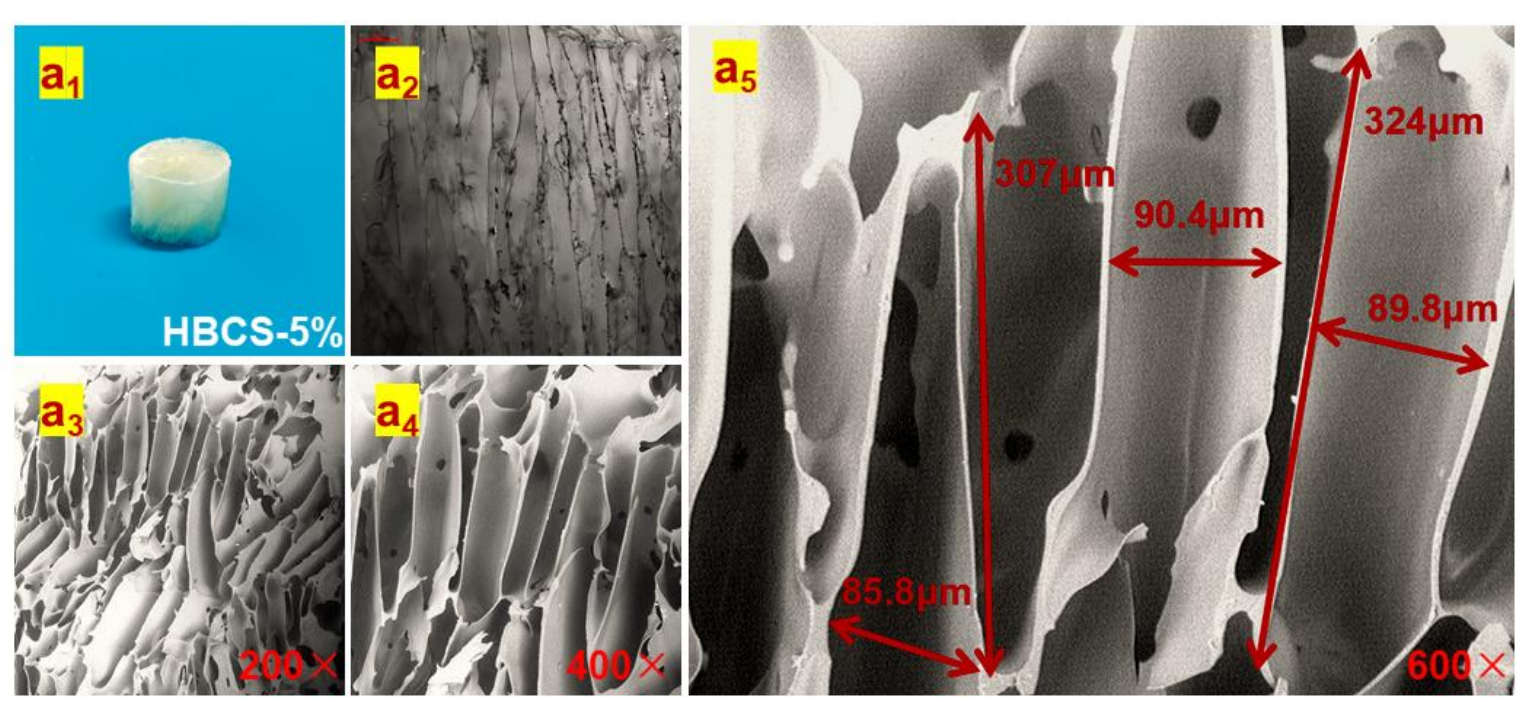

(b)

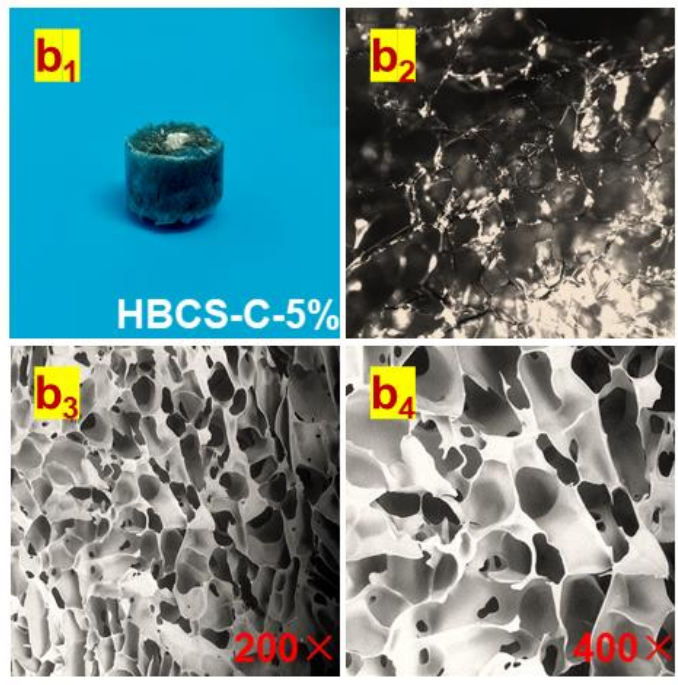

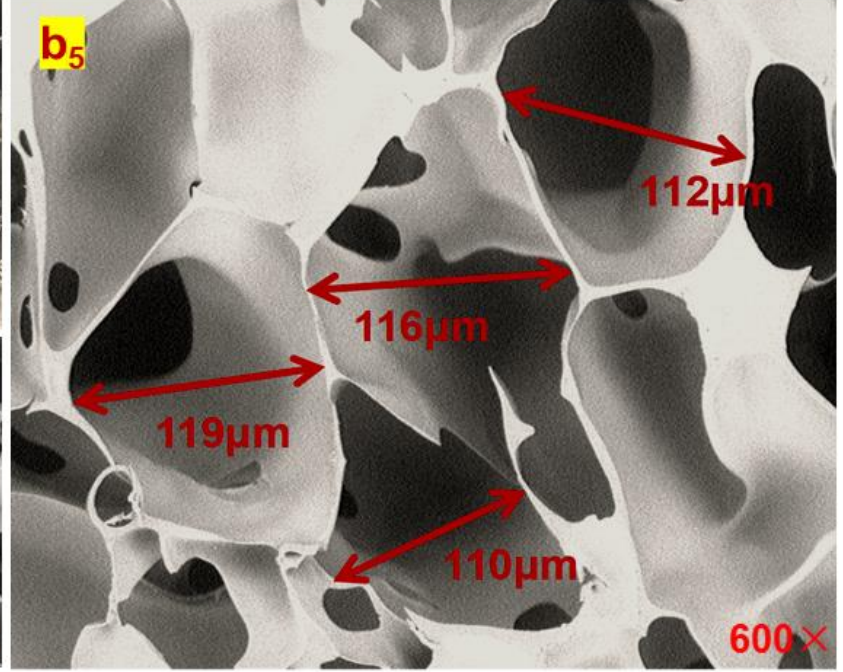

Figure S3. Micro-morphology of $5 \mathrm{wt} \%$ (a) HBCS and (b) HBCS-C hydrogels. a was the morphology diagram of HBCS hydrogel after lyophilization, $\mathrm{a}_{2}$ was the stereo-microscope image of HBCS hydrogel, $\mathrm{a}_{3}-\mathrm{a}_{5}$ were the SEM images of HBCS hydrogel at different magnifications $(200 \times, 400 \times$, and $600 \times)$; $b_{1}$ was the morphology diagram of HBCS-C hydrogel after lyophilization, $b_{2}$ was the stereo-microscope image of HBCS hydrogel, $b_{3}$ $b_{5}$ were the SEM images of HBCS-C hydrogel at different magnifications $(200 \times, 400 \times$, and $600 \times)$. 


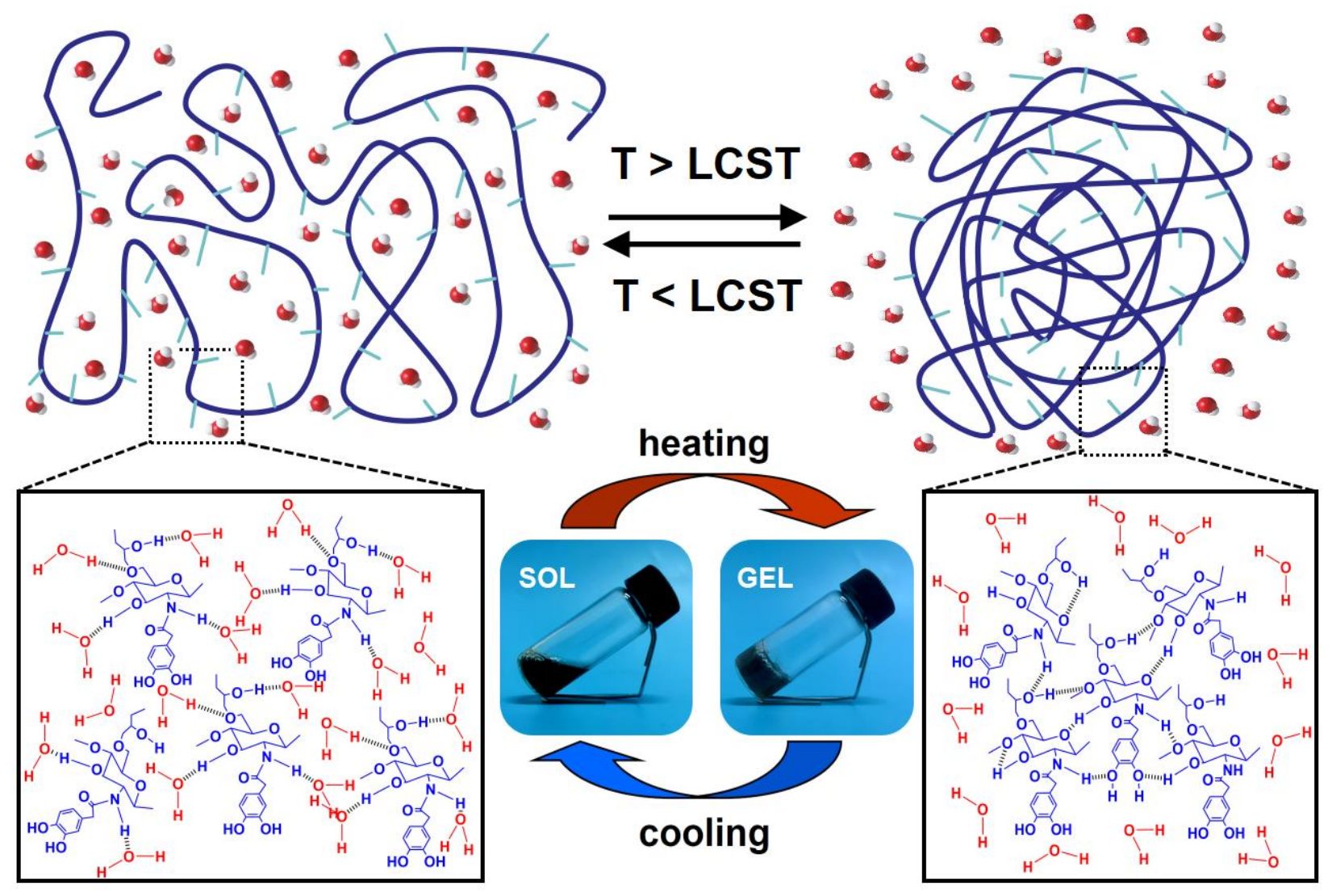

Figure S4. Schematic representation of thermoresponsive mechanism of HBCS-C hydrogel. The thermoresponsive ability of HBCS-C hydrogels was based on the changes of intramolecular/intermolecular hydrogen bonds and hydrophobic interactions. At room temperature, intramolecular and intermolecular hydrogen bonds existed between hydroxybutyl groups, water molecules, and chitosan backbones, hindering the aggregating of HBCS-C molecules. As the temperature rose to the lower critical solution temperature (LCST), the decreasing intermolecular hydrogen bonding actuated the water molecules around the HBCS-C molecules to move away. Meanwhile, it leaded to the dewatered hydrophobic chains entangle each other and form solid matrix. 


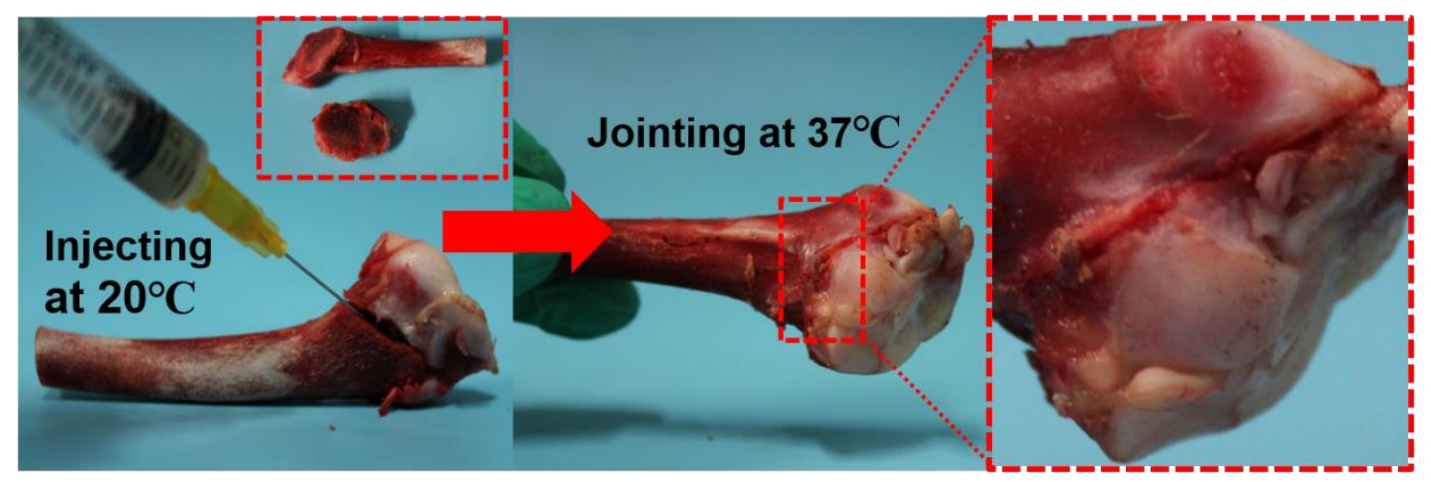

Figure S5. Tissue adhesive behavior of HBCS-C hydrogels, which showed two chicken bones were jointed by HBCS-C hydrogel (5 wt \%) due to its well-performed adhesion.

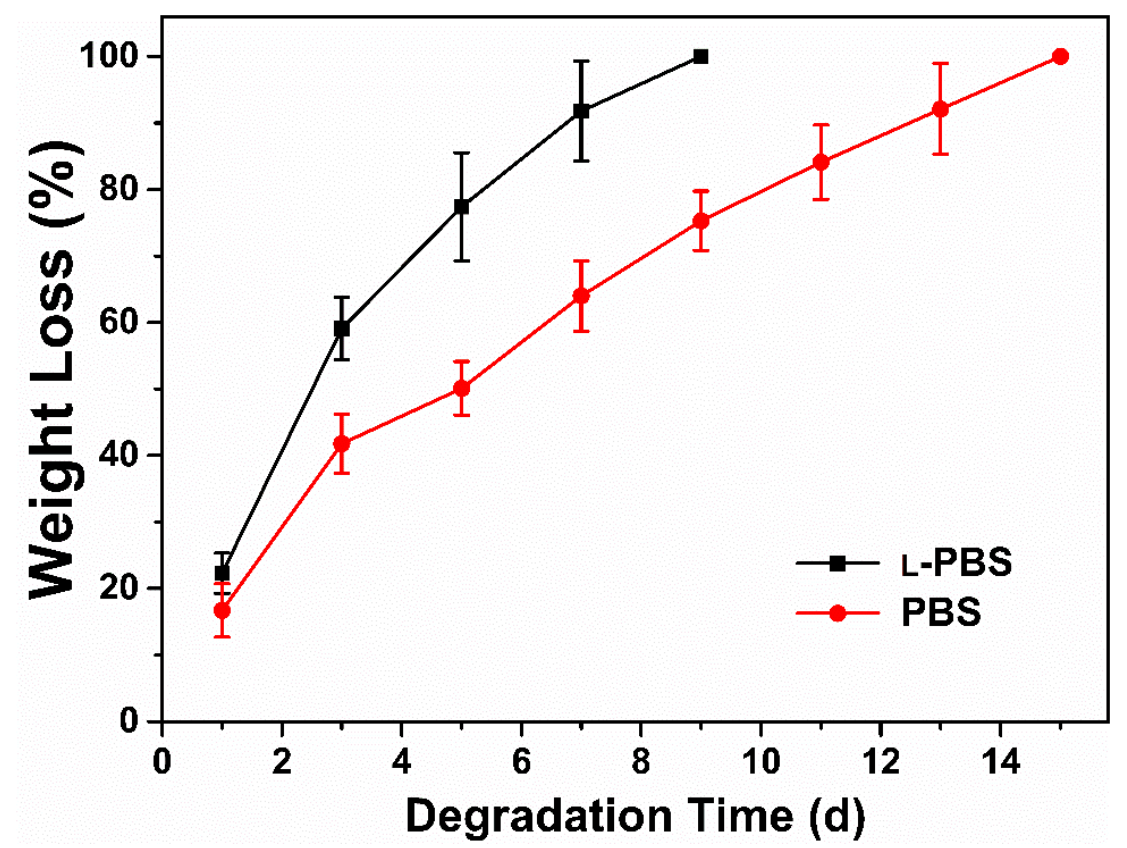

Figure S6. Degradation in vitro of HBCS-C hydrogels in PBS and L-PBS (1 mg/mL lysozyme in PBS) with stirring magneton at $37^{\circ} \mathrm{C}$. Each value represented the mean $\pm \mathrm{SD}(\mathrm{n}=3$ per each group). 
Table S1. Grafting ratio and Mv of HBCS-C at different molar feed ratios of DOPAC to HBCS (the DS of CS and $\mathrm{HBCS}=0.969$ ).

\begin{tabular}{lccc}
\hline Sample & $\begin{array}{c}\text { Molar feed ratio of } \\
\text { DOPAC to HBCS }\end{array}$ & $\begin{array}{c}\text { Grafting ratio of } \\
\text { catechol groups }\end{array}$ & $\begin{array}{c}\text { Viscosity-average } \\
\text { molecular weight (kDa) }\end{array}$ \\
\hline CS & - & - & $1159.9 \pm 9.6$ \\
\hline HBCS & $1: 1$ & - & $836.8 \pm 10.3$ \\
\hline & $2: 1$ & $18.1 \%$ & $892.8 \pm 11.7$ \\
HBCS-C & $3: 1$ & $23.5 \%$ & $918.4 \pm 10.4$ \\
& & & $931.7 \pm 8.6$ \\
\hline
\end{tabular}

